\title{
PRACTICAL MEANS OF PREOPERATIVE DIAGNOSTICS OF PRIMARY FALLOPIAN TUBE CANCER
}

D0l:10.36740/WLek202102120

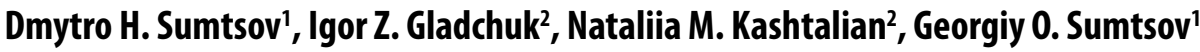 \\ 'SUMY STATE UNIVERSITY, SUMY, UKRAINE \\ 20DESSA NATIONAL MEDICAL UNIVERSITY, ODESA, UKRAINE
}

\begin{abstract}
The aim: To analyze contemporary practical means to improve diagnostics of primary fallopian tube cancer.

Materials and methods: Authors analyzed specifics of clinical signs and anamnesis in 152 PFTC patients. Diagnostic capacity of cytological analysis of pathologic vaginal discharge, $X$-ray contrast methods of examination, sonography, tumor markers, and computed tomography was studied. 0 wn results of PFTC diagnostics using different methods and world practice using MRI, PET-CT and laparoscopy were discussed.

Results: Using own observations authors conclude that clinical analysis and complex use of the listed methods allows to mainly determine high risk group patients and set correct preoperative diagnosis in $35 \%$ and preliminary diagnosis in $20 \%$ of PFTC patients.

Conclusions: Complex examination allows to recognize primary fallopian tube cancer on preoperative stage and to avoid inadequate surgical interventions in majority of PFTC patients.
\end{abstract}

KEY WORDS: primary fallopian tube cancer, complex examination, preoperative diagnostics

\section{INTRODUCTION}

According to literature data and own observations, primary fallopian tube cancer (PFTC) amounts to no less than $1.5-1.9 \%$ of female genital cancers and $4-6 \%$ of cancers of uterine appendages. However, the true frequency of fallopian tube cancer is likely significantly higher, given that up until now, if neighboring organs are involved, this pathology is often considered being ovarian cancer [1 - 4]. Besides, epidemiological studies show increase in PFTC morbidity. Thus, from 2010 to 2014 morbidity in the USA increased 4-fold [5,6]. Despite the improvement of diagnostic capacity of the contemporary methods of examination, accuracy of preoperative diagnostics of PFTC even in specific oncology centers still varies between $0 \%$ and $12-15 \%[2,4,7,8]$. Diagnostic mistakes delay detection and complicate treatment of these patients. Thus, finding means of improvement of preoperative diagnostics of PFTC is a pressing medical issue.

\section{THE AIM}

Aim of the study was to conduct retrospective analysis of own long-term clinical observation and results of additional examination methods for further improvement in detection and effectiveness of preoperative diagnostics of PFTC, to review literature data and make practical conclusions.

\section{MATERIALS AND METHODS}

For the analysis we chose 152 PFTC patients with conclusive case histories. Age of the patients was between 34 to 78 years (mean age $-54.5 \pm 4.9$ years). Age group from 46 to 65 years old contained $65 \%$ of PFTC cases. Diagnostic capacity of cytological analysis of pathologic vaginal discharge, X-ray contrast methods of examination, sonography, tumor markers, and computed tomography was studied. Own results of PFTC diagnostics using different methods and world practice using MRI, PET-CT and laparoscopy were discussed.

\section{RESULTS}

According to anamnesis and surgical revision data, around $70 \%$ of PFTC patients had pelvic inflammatory disease (PID) and $49 \%$ had infertility. All patients underwent surgical treatment and had diagnoses confirmed by histopathology. The majority of cases were serous carcinomas of different structure and differentiation (116 (76.3\%)), second most common tumors were endometrioid (19 $(12.5 \%))$ and undifferentiated carcinomas (12 (7.9\%)). Singular findings of clear cell, mucinous, and squamous carcinomas amounted to only $3.3 \%$ of cases.

In the study were analyzed specifics of clinical signs in primary fallopian tube cancer patients, and data of additional methods of examination in these patients on preoperative stage. 
Previously performed analysis established that the reasons for delayed diagnostics of PFTC are mainly ignorance of clinical specifics of the tumor and insufficient cancer alert, and less - delayed seeking of medical care, hidden or atypical disease course [4].

The main clinical symptoms in most patients $114(75$ $\pm 3.5 \%)$ in the analysis, were the presence of secretions similar to lymphorrhea, bloody discharge in pre- and postmenopause, as well as the most typical sign of PFTC - hydrops tubae profluens $20(13.2 \pm 2.7 \%)$.

After seeking medical help during the bimanual examination, atypical changes of the appendages were palpated, which were confirmed during sonography, but could be interpreted as pathology of the uterus. Several women had a clinical picture of acute abdomen, which required emergency surgery in non-oncology departments. Diagnosis of PFTK was established only after histological examination.

Examination of women with adnexal masses, with or without suspicion of PFTC, on preoperative stage includes different additional investigational methods. One of the most available additional methods is cytological examination of pathological uterine discharge.

To make a preoperative diagnosis, we make extensive use of X-ray contrast techniques (hysterosalphingography (HSG) and bicontrast HSG), which often allow PFTC to be diagnosed at an early stage. According to the analysis, the correct diagnosis was in 62 ( $82 \pm 4 \%$; CI: 71-90) patients, in contrast to the use of ultrasound scanning. Only in 2 (13.3\%) cases USS showed a typical picture of PFTK.

An additional method in the complex diagnosis of PFTK are tumor markers, namely CA-125. Although its diagnostic accuracy is very controversial $[2,3,8]$. In our analysis, we concluded that CA-125 is not accurate enough in the early stages, but is a significant early marker of cancer recovery. Diagnostic laparoscopy should be considered as an intraoperative diagnosis. However, its advantages, features and disadvantages require further in-depth study.

\section{DISCUSSION}

Many authors studying primary fallopian tube cancer report difficulties of its preoperative diagnostics: some of them blame oligosymptomatic nature of the disease, others blame low specificity of the symptoms [2,3,4,7]. Diagnostics of the disease is challenging even during laparotomy, which is proven by reports of $30-50 \%$ of incorrect diagnoses during surgery $[4,9,10]$. Most often it happens in patients in the early stages of the disease who undergo surgery in non-oncological profile hospitals without suspicion of malignization, which leads to nonradical surgery and delay, or even sometimes absence, of adequate treatment. Up-to-date some authors consider diagnosis of PFTC as an intraoperative finding, others - as pathoanatomical rather than clinical diagnosis $[2,11,12]$. Even in a specialized oncological institution correct diagnoses of PFTC during surgery were made only in $47.5 \%$ cases [7].

$114(75 \pm 3,5 \%)$ patients with PFTC complained of different kind of pathological discharge from sexual pathways.
In most cases discharge were liquid and opalesque, of yellowish or amber colour, mainly lymphorrhea-like, with constant or periodic blood admixture noticed in up to half of those patients. The second most common discharge type were bloody spotting in postmenopause, beginning bloody spotting in premenopause was commonly considered being an acyclic uterine bleeding. $20(13,2 \pm 2,7 \%)$ patients had the most typical sign of PFTC - hydrops tubae profluens, a condition when profuse watery vaginal discharge happens during colicky pain episode which leads to pain relief and decrease in size or even disappearance of a previously palpated tubo-ovarian mass. More rarely patients (5.2\%) experienced vaginal discharge of variable color or yellowish discharge with purulent admixture. Typical for all types of vaginal discharge in PFTC is their common connection with pain syndrome, persistent increase and ineffectiveness of different treatment measures including uterine diagnostics curettage. Rarely discharge are mild and can be diagnosed only on colposcopy or during sonography, when fluid in uterine cavity is found. $107(70,4 \pm 3,7 \%)$ patients with PFTC complained of pain. Around a third of them had dull sometimes colicky pain in the lower abdomen on an affected side, rarer like renal or tubal colic. In cases of aggravation of concomitant chronic inflammatory process, torsion of affected tube or its perforation some patients $(6(3,9 \%))$ developed "acute" abdomen. In cases of disseminated tumors involving neighboring organs patients complained of different types of pain and other signs, including weakness, fatigue, fever, discomfort and bloating in abdomen, dysuria, etc.

According to patients' histories and intraoperation revision data, around $70 \%$ of patients with PFTC had pelvic inflammatory disease and $49 \%$ complained of infertility.

Upon seeking medical care, 116 (76,3 $33,4 \%)$ PFTC patients already had anatomical changes of internal genitals, which were revealed on bimanual examination. In more than half of the patients enlargement of appendages were palpated as ovoid, sausage-like or irregular formation of uneven, more often rubber elastic consistency, sometimes mobile and almost painless. Almost every fifth patient had unclear changes in appendages, which were interpreted as pathology of uterus. As opposed to ovarian cancer, implantation metastases and tumor infiltrates in rectouterine pouch are not common in PFTC. All this signs and clinical findings, while grouped into symptom complexes, created variety of clinical courses of PFTC, often masked as other, no less dangerous diseases.

It is worth to mention that $10(6,5 \pm 1,9 \%)$ PFTC patients prior to diagnosis considered themselves healthy and had no complaints. In them the disease was found on routine examination, including 4 cases found on routine sonography or while diagnosing another pathology.

However, according to our observations, 25 (16,4 $43,0 \%)$ patients had typical for PFTC classic clinical triad decribed by Latzko in 1916 [4,12]. It includes waterish, lymph like, discharge related to pain syndrome, often hydrops tubae profluens, and palpated (or found on ultrasonography) characteristic masses in the areas of adnexa. 
Quite typical clinical picture was present in $23(15,1 \pm 3,3 \%)$ patients. They had one or two typical symptoms and other less significant signs (anamnesis, age, treatment ineffectiveness), which allowed to suspect PFTC and use more informative additional diagnostic methods.

PFTC, clinically proceeding similar to endometrial cancer, was found in $23(15,1 \pm 2,9 \%)$ patients. Majority of those patients underwent, sometimes even repeatedly, dilation and curettage, however, groups of tumor cells were found in 2 cases and typical endometrial cancer - in 1 case. The latter was endometrial cancer synchronous to fallopian tube cancer (histopathological and immunohistochemical analyses revealed serous papillary adenocarcinoma in uterine tube and highly differentiated endometrioid adenocarcinoma in uterus). $28(18,4 \pm 3,1 \%)$ patients were diagnosed with different adnexal masses on ultrasonography, mostly thought to be ovarian cysts or hydrosalphinx, suspicion of malignancy was stated if papillary outgrowth was present. $12(7,9 \pm 2,1 \%)$ PFTC patients were hospitalized with initial diagnosis of leiomyoma of uterus, while a part of them really had it, and some were observed and treated previously. There were cases, where typical for PFTC sonographic picture with normal ovaries was considered to be a subserosus pedunculated fibroid with signs of necrosis.

Clinical picture of "acute" abdomen was present in 6 $(3,9 \pm 1,5 \%)$ patients, all of them had emergency surgery in non-oncology departments. Half of those patients had incomplete radical operations and diagnosis of PFTC was established only after histopathological study.

$14(9,2 \pm 2,3 \%)$ PFTC patients had prolonged treatment for pelvic inflammatory disease, often with temporary subjective improvement. In them PFTC imitated PID, or PID was a concomitant process. Diagnostically challenging situation was present in $9(5,9 \pm 1,9 \%)$ young women, in whom PFTC masked as abnormal uterine bleedings, and in $2(1.3 \pm 0,9 \%)$ PFTC patients an extragenital tumor was suspected prior to laparotomy.

Examination of women with adnexal masses, with or without suspicion of PFTC, on preoperative stage includes different additional investigational methods. One of the most available additional methods is cytological examination of pathological uterine discharge. Probability of receiving positive result in patients with discharge is $75 \%$. Although according to literature data, accuracy of cytological results varies between $0 \%$ and $40 \%$. It is explained by hardship of obtaining cytological sample of sufficient quality and quantity [13-16]. We specifically examined 95 PFTC patients using different methods of sampling (vaginal and cervical smears; uterine aspirate; discharge on cervical cap; discharge sampled on hysterosaplingography). Different methods provided accurate preoperative results in $11 \%-65 \%$ of cases. The least informative were routine vaginal and cervical smears. That is why we recommend to use a combination of sampling methods [17].

Starting from the 70s and 80s we have widely used X-ray contrast methods (hysterosalphingography (HSG) and bicontrast HSG). Among 76 PFTC patients examined by cytology and X-rays, 44 (58 $\pm 6 \%$; CI: $46-69)$ patients received accurate preoperative diagnosis, together with suspicious for PFTC cases, the correct diagnosis was present in 62 (82 $\pm 4 \%$; CI: 71-90) patients. HSG should still be included into diagnostic spectrum. For instance, US authors consider that this method allows correction between MRI and sonography. In 2005 visual atlas of HSG was published $[4,18]$. We still periodically use HSG, and this method, used together with other methods, often allows for early stage PFTC diagnostics [17].

Ultrasound scanning (USS) is often used in gynecology for diagnostics of different pathology and sometimes as screening due to patient's wish. In 2006 we have originally analyzed USS results on preoperative stage in 15 PFTC patients. Only in $2(13,3 \%)$ cases USS showed typical picture of PFTC, and experienced clinician could suspect and state correct diagnosis. Many authors report the similar [18,20]. But technical progress leads to improvement in diagnostics, especially after introduction of transvaginal and doppler energetic scanning. During the last decade we performed USS on preoperative stage in 43 PFTC patients. Out of them 8 (19 $\pm 6 \%$; CI: 8-33) patients received accurate or preliminary diagnosis of PFTC, and 9 (21 $\pm 6 \%$; CI: $11-37)$ patients had quite typical clinical picture, suspicious for PFTC. Therefore, only sonography already allowed for accurate clinical diagnosis in $17(40 \pm 8 \%$; CI: 25-56) patients. $8(18,6 \pm 6 \%)$ patients from the same group received ultrasonic diagnosis of ovarian cancer, $7(16,3 \pm 5,6 \%)$ patients received diagnosis of cyst or papillary cyst of ovaries, 8 $(18,6 \pm 6 \%)$ patients - of sactosalphinx or other adnexal masses, and only in 3 patients no pathology was found. Alas, ultrasonic signs of PFTC are not always specific and can imitate tubal pregnancy, tuboovarian abscesses, tuberculosis of adnexa or another pathology $[7,19,21]$.

Literature data suggest that magnetic resonance imaging (MRI) and positron emission tomography (PET) are often used for PFTC diagnostics [1,22,23]. According to some authors these methods not only visualize a tumor and its structure, but unlike USS, allow to recognize fine details of contrast, of hydrophilic and chemical specifics of tissues, to identify tumor infiltrates, their borders and metastases in regional lymph nodes [23-25]. There are even suggestions to include MRI into FIGO guidelines as a compulsory diagnostic procedure for PFTC [24].

We were unable to find current literature data on preoperative diagnostics of PFTC using computed tomography (CT). Out of our 12 PFTC patients who underwent CT, and 6 patients who underwent MRI, only 1 patient received clear description (on spiral CT) of a "109x47x54 mm mass in the projection of a left fallopian tube", which together with clinical and USS data allowed to state accurate diagnosis on a preoperative stage. Examination results in other patients were not sufficiently informative.

Tumor markers are also used in complex diagnostics of PFTC. Literature data mainly suggests CA-125, but reports of its diagnostic accuracy are very contradictory $[2,3,8]$. According to many separate observations and our data, levels of CA-125 are usually normal or dubious in the early stages of PFTC, especially if concomitant chronic diseases are present $[4,11,23]$. After radical surgery or chemotherapy, 

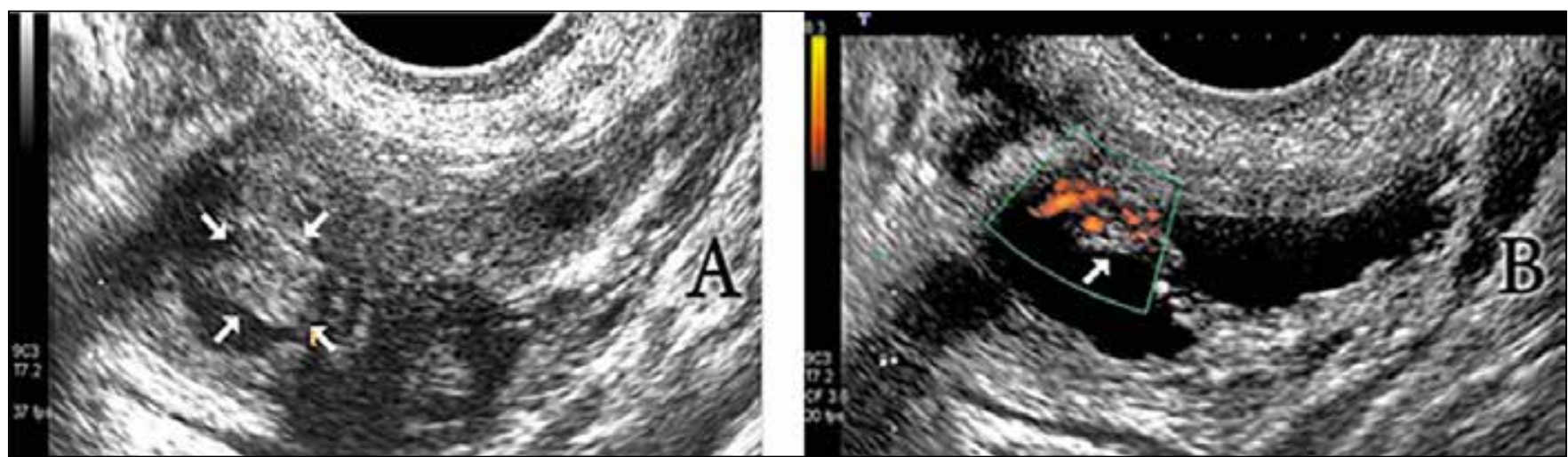

Fig. 1. Sonography scans of the patient.

A - multiple papillary outgrowth on the inner wall of fallopian tube (pointed by arrows).

B - on Doppler energetic (colorful) scanning presence of moderate intensity hypervascularisation with increased speed and decreased resistance in the same area of fallopian tube (pointed by arrow).

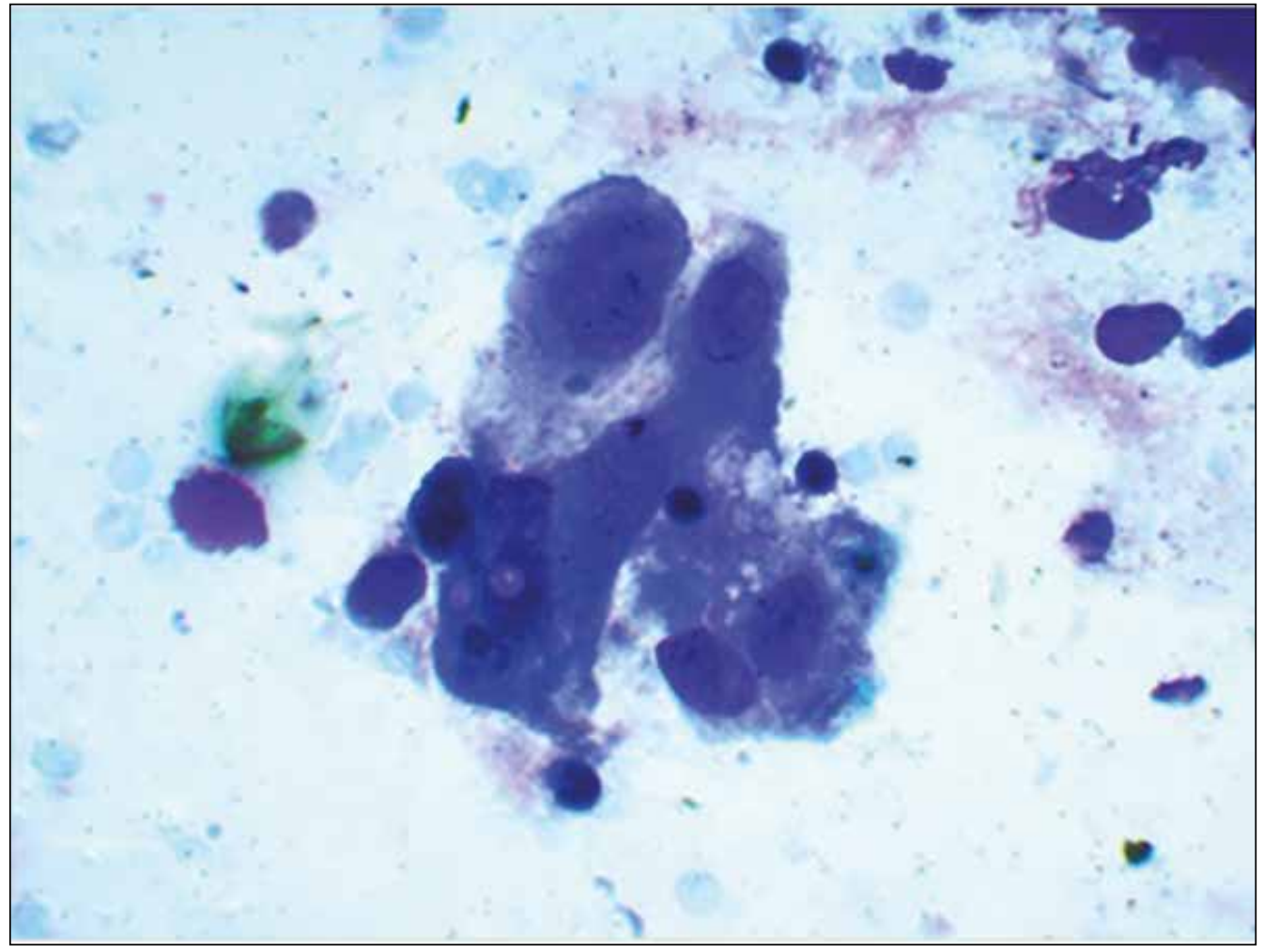

Fig. 2. The patient's uterine cavity aspirate. Photo of micropreparation. Romanowsky-Giemsa staining. x400 enlargement. level of CA-125 normalizes, and increases again in cancer reactivation $[8,11]$. This allows for 3 months earlier diagnostics of disease recurrence compared to clinical-laboratory or X-ray methods $[3,8,11]$. We analyzed results of CA-125 testing on different stages of diagnostics and treatment in 30 PFTC patients, our conclusion is that CA-125 is not sufficiently accurate on the early stages, but is a significant early marker of a cancerous process reactivation [4].

\section{CASE REPORTS}

In order to demonstrate practical means of PFTC diagnostics, we would like to provide one of our latest case reports.

Patient B, 49 years old, addressed a gynecologist with complaints of lower abdominal pain. On bimanual exam- ination - slight pain on shifting of uterus; on colposcopy - a drop of amber liquid in cervical canal, lymphorrhea was suspected. Immediately after doctor performed uterine aspiration for cytological test, patient was referred to USS. Sonography revealed bilateral hydrosalpinx, on the left with papillary structures $6.2 \times 2.9 \mathrm{~mm}$ with strong blood flow of increased speed and low resist; impression of probably malignant neoplasm in distal part of left fallopian tube (Fig.1).

Cytological analysis of uterine aspirate revealed elements of glandular cancer (Fig.2). Other examinations results: CT - small fibroids in uterus, ovaries are normal, left side hydrosalpinx; CA-125 - 14,69 U/ml (normal $\leq 35,0 \mathrm{U} / \mathrm{ml}$ ); HE-4 - 104 pmol/1 (normal $\leq 74,3 \mathrm{pmol} / \mathrm{l}$ ). Preoperative diagnosis: left sided PFTC.

Patient underwent radical hysterectomy with adnexa, pelvic and lower lumbar lymph nodes, and greater omentum resec- 
tion. Histopathological analysis: serous adenocarcinoma of left fallopian tube without serous layer invasion, metastases in right fallopian tube and right upper iliac lymph nodes. Final diagnosis: Primary fallopian tube cancer T2A N1 Mo.

A group of cells of mucous epithelium with considerable polymorphism of nuclei. Nuclei contain 1-2 nucleoli, fine chromatin.

Diagnostic laparoscopy should be regarded as intraoperative diagnostics. According to R.Wenzl at al. the odds of finding PFTC in general laparoscopic practice is 1 per 3687 laparoscopies or $0.028 \%$ [4]. Literature search showed only case reports on diagnostic laparoscopy in PFTC, without detailed elaboration of diagnostic specifics $[23,27,28]$. Considering prolonged 'closed nature' of PFTC and masked progression as hydro- or hematosalpinx, there are very low chances of recognizing on laparoscopy without histopathological analysis a primary fallopian tube cancer without peritoneal spread. Spread of cancer cells from punctured or reopened fallopian tube to pelvic cavity considerably worsens patient's prognosis. Patrick F. Timmins et al. report decrease of 10-years survival from $58 \%$ to $7 \%$ if cancer cells are found in abdominal cavity [29]. Indications, advantages and specifics of laparoscopic diagnostics of PFTC should be studied further.

\section{CONCLUSIONS}

The current analysis of anamnestic and clinical specifics in PFTC patients proves that proper oncologic alertness and complex focused use of the methods, mentioned in the article, allows for accurate suspicion and correct preoperative diagnostics in PFTC patients, leading to referral to specialized medical centers. During years 2015-2019 there were 23 cases of PFTC in our region. Out of them 3 patients were operated in non-specialized hospitals, including 1 patient operated in another region. In 2 of them oncologic process wasn't recognized on operation. The remaining 20 out of total 23 PFTC patients had complex examination and surgery in regional clinical oncologic dispensary. Accurate preoperative diagnosis was set in $7(35 \pm 11 \%)$ patients, preliminary diagnosis - in $4(20 \pm 9 \%)$ patients. Three $(15 \pm 8 \%)$ patients has preoperative diagnosis of endometrial cancer, because elements of the tumor were found on diagnostic dilation and curettage. One of them had simultaneously endometrial cancer and PFTC. In the other 6 $(30 \pm 10 \%)$ patients adnexal masses were recognized as ovarian cancer or papillary cyst suspicious for malignancy. Mainly, PFTC was diagnosed on preoperative stage in the major half of patients. Though, we must stress that correct diagnoses were set mainly basing on the clinical signs of the disease.

We consider the following important:

- if pathological discharge are present, especially in women of high risk groups, several methods of cytological sampling should be used at once;

- if adnexal mass is visualized on sonography, further examination should be carried out using transvaginal scanning in energetic Doppler mode with special attention to the character of contents, capsule, structure of the mass, and especially blood flow;
- if necessary, use MRI, PET-CT and X-rays contrast methods of diagnostics;

- puncture of is adnexal masses is unacceptable, including during laparoscopy;

- if PFTC is found on operation and there is no possibility to invite oncogynecologist, the patient should be transferred to oncogynecology department directly after surgery. Detailed description of revision of abdominal and retroperitoneal structures should be provided for adequate staging and determination of necessity of radical surgery or other methods of treatme

\section{REFERENCES}

1. Winarto $H$., Fernando D. Identifying features of primary fallopian tube carcinoma using magnetic resonance imaging. Int Med Case Rep J. 2017;10:213-217. doi: 10.2147/IMCRJ.S135836.

2. Gungorduk K., Ertas I.E., Ozdemir A. et al. Prognostic Significance of Retroperitoneal Lymphadenectomy, Preoperative Neutrophil Lymphocyte Ratio and Platelet Lymphocyte Ratio in Primary Fallopian Tube Carcinoma: A Multicenter Study. Cancer Res Treat. 2015;47(3):4808. Doi: $10.4143 /$ crt.2014.058.

3. Pectasides D., PectasidesE., Economopoulos T. Fallopian Tube Carcinoma: A Review.The Oncologist.2006;11(8):902-912.

4. Sumtsov G., Sumtsov D. [Primary fallopian tube cancer:Первичный рак маточных труб: Monograph]. Sumy: Sumy State University. 2015:229. (In Russian).

5. Trabert B., Coburn T., Mariani A. et al. Reported Incidence and Survival of Fallopian Tube Carcinomas: A Population-Based Analysis From the North American Association of Central Cancer Registries. J Natl Cancer Inst. 2018;110(7):750-7. Doi: 10.1093/jnci/djy078

6. Liao C.I., Chow S., Chen L.M. et al. Trends in the incidence of serous fallopian tube, ovarian, and peritoneal cancer in the US. Gynecol Oncol. 2018;149(2):318-323. Doi: 10.1016/j.ygyno.2018.01.030.

7. Bulanov M., Pavlova N., Gorkina 0., Voronova M. [Ultrasound diagnostics of primary fallopian tube cancer]. Ultrasound and functional diagnostics. 2014; 2:42-56. (In Russian).

8. Eken M., Temizkan 0., Kaygusuz E.I. Primary carcinoma of the fallopian tubes: Analysis of sixteen patients. Turkish J ObstGynec. 2015;12(2):83-88. D0I: 10.4274/tjod.67355 PMID: 28913049.

9. Horng H.C., Teng S.W., Huang B.S. et al. Primary fallopian tube cancer: domestic data and up-to-date review. Taiwan J Obstet Gynecol. 2014; 53(3):287-92. doi: 10.1016/j.tjog.2014.07.003.

10. Hei-Yu Lauab, Yi-Jen Chenab, Ming-ShyenYenabet al. Primary fallopian tube carcinoma: A clinicopathologic analysis and literature review. Journal of the Chinese Medical Association. 2013;76(10): 583-587. https://doi.org/10.1016/j.jcma.2013.06.010.

11. Akkaya E., Sanci M., Nur Kulhan G.et al. Prognostic factors of primary fallopian tube carcinoma Contemp Oncol (Pozn). 2018; 22(2): 99-104. . doi: 10.5114/w0.2017.69590.

12. Mladenović-Segedi L.Primary fallopian tube carcinoma. Med Pregl. 2009;62(1-2):31-6.

13. Ural U.M., Balik G., Tekin Y.B., Sehitoglu I. et al. Primary fallopian tube carcinoma diagnosed preoperatively by cervical smear. Ann Saudi Med. 2014;34(5):444-446. D0l: 10.5144/0256-4947.2014.444.

14. Otsuka I., Kameda S., Hoshi K. Early detection of ovarian and fallopian tube cancer by examination of cytological samples from the endometrial cavity. Br J Cancer. 2013; 109(3): 603-609. doi: 10.1038/bjc.2013.402. 
15. Buskmiller C., Cline B., Xynos F. Medical diligence uncovers fallopian tube cancer after abnormal Pap test. BMJ Case Rep. 2019;12(7): e229762. doi: $10.1136 / \mathrm{bcr}-2019-229762$.

16. Rubeša-Mihaljevićm R., Verša Ostojić D., Dinter M., ŠtembergerPapić S. et al. The role of cytology in preoperative assessment od primary fallopian tube carcinoma: case report. Medicina Fluminensis. 2016;52(3):415-420.

17. Sumtsov G.A., Hyriavenko N.I., Starkiv M.P. et al. Primary fallopian tubes cancer: incidence, problems of diagnosis and treatment. ATJ, 2019;3:123-129.

18. Ledbetter K.A., Shetty M. Hysterosalpingography: an imaging Atlas with cross-sectional correlation. Myers Abdom. 2015;40(6):1721-32.

19. Balaya V., Metzger U., Lecuru F. Ultrasonographic features in the preoperative diagnosis of primitive fallopian tube carcinoma. J Gynecol Obstet Biol Reprod. Paris .2016; 45(1):11-20.

20. Ludovisi M., De Blasis I., Virgilio B. et al. Imaging of gynecological disease (9): clinical and ultrasound characteristics of tubal cancer. Ultrasound. Obstet. Gynecol. 2014; 43(3):328-35.

21. Hereter L., Pascual M., Tresserra F. et al. Ultra_sonographic features of Fallopian tube cancer. Ultrasound Obstet. Gynecol. 2012;40(1):51-54.

22. Kitai S., Kiyokawa T., Tanaka Y.0., Onoue K. et al. MRI findings for primary fallopian tube cancer: correlation with pathological findings. Jpn J Radiol. 2018;36(2):134-141. doi: 10.1007/s11604-017-0705-0.

23. Takano M., Kagawa Y., Murata S., Fujiwara M. et al. Fallopian tube cancer incidentally diagnosed during laparoscopy for metastatic adenocarcinoma of unknown primary lesion. Gynecol Minimal Invasiv Therapy. 2016; 5(4):166-169. doi.org/10.1016/j.gmit.2015.06.007.

24. Ma X., Huang X., Chen C., Ding Y. A Preliminary Report Requiring Continuation of Research to Confirm Fallopian Tube Adenocarcinoma: A Non-Experimental, Non-Randomized, Cross-Sectional Study. Med Sci Monit. 2018; 24:5301-5308.

25. Gomes F.V., Dias J.L. Lucas , Cunha T.M. Primary fallopian tube carcinoma: review of MR imaging findings. Insights Imaging. 2015; 6(4):431-439. doi: 10.1007/s13244-015-0416-y.

26. Cai S.Q., Ma F.H., Qiang J.W., Zhao S.H. et al. Primary fallopian tube carcinoma: correlation between magnetic resonance and diffuse weighted imaging characteristics and histopathologic findings. J Comput Assist Tomogr. 2015;39(2) :270-5. doi: 10.1097/ RCT.0000000000000178.

27. Chen Y., Ling C., Bian C. Port-site metastasis as a primary complication following diagnostic laparoscopy of fallopian tube carcinoma: A case report. Medicine (Baltimore). 2018; 97(26):e11166. doi: 10.1097/ MD.0000000000011166.
28. Madhuri T.K., Tailor A., Butler-Manuel S. Laparoscopic interval surgery for stage 4 primary fallopian tube carcinoma - a case report. Gynecol Surg. 2009; 6: 371. https://doi.org/10.1007/s10397-009-0507-1.

29. Timmins P., Kanbour A., Price F. Predictors for survival in fallopian tube carcinoma. Priory Medical Journals. 2020. http://www.priory.com/med/ fallopian.htm

Improvement of methods of prevention, diagnosis and treatment of disease of the reproductive system of a woman using the latest medical and molecular genetic technologies. № 0117 U007494.

\section{ORCID and contributionship:}

Dmytro H. Sumtsov : 0000-0001-5143-6902 A, B, C, D

Igor Z. Gladchuk : 0000-0003-2926-4125 D, E, F

Nataliia M. Kashtalian: 0000-0003-1386-366 ${ }^{D}$

Georgiy O. Sumtsov: 0000-0002-7422-9399 A, B, C, D

\section{Conflict of interest:}

The Authors declare no conflict of interest.

\section{CORRESPONDING AUTHOR} Igor Z. Gladchuk

National Medical University

9 Pastera St, 65000 0dessa, Ukraine

tel: +380676547000

e-mail:igor.gladchuk@gmail.com

Received: 09.08 .2020

Accepted: 05.01.2021

A - Work concept and design, B - Data collection and analysis, C - Responsibility for statistical analysis, D-Writing the article, $\mathbf{E}$ - Critical review, $\mathbf{F}$ - Final approval of the article 